\title{
Hausdorff Distance evaluation of orthodontic accessories' streaking artifacts in 3D model superimposition
}

\section{José Rino Neto(a) \\ Fernando Penteado Lopes da \\ Silva ${ }^{(a)}$ \\ Israel Chilvarquer(b) \\ João Batista de Paiva ${ }^{(a)}$ \\ Angélica Maria Hernandez ${ }^{(\text {b) }}$}

(a) Department of Orthodontics, School of Dentistry, Univ of São Paulo, São Paulo, SP, Brazil.

(b) Department of Stomatology, School of Dentistry, Univ of São Paulo, São Paulo, SP, Brazil.
Declaration of Interests: The authors certify that they have no commercial or associative interest that represents a conflict of interest in connection with the manuscript.

Corresponding author: Fernando Penteado Lopes da Silva E-mail: ferpenteado@usp.br

Submitted: Mar 02, 2012 Accepted for publication: Jul 01, 2012 Last revision: Jul 18, 2012

\begin{abstract}
The aim of this study was to determine whether image artifacts caused by orthodontic metal accessories interfere with the accuracy of 3D CBCT model superimposition. A human dry skull was subjected three times to a CBCT scan: at first without orthodontic brackets (T1), then with stainless steel brackets bonded without (T2) and with orthodontic arch wires (T3) inserted into the brackets' slots. The registration of image surfaces and the superimposition of 3D models were performed. Within-subject surface distances between T1-T2, T1-T3 and T2-T3 were computed and calculated for comparison among the three data sets. The minimum and maximum Hausdorff Distance units $(\mathrm{HDu})$ computed between the corresponding data points of the T1 and T2 CBCT 3D surface images were 0.000000 and $0.049280 \mathrm{HDu}$, respectively, and the mean distance was $0.002497 \mathrm{HDu}$. The minimum and maximum Hausdorff Distances between T1 and T3 were 0.000000 and $0.047440 \mathrm{HDu}$, respectively, with a mean distance of $0.002585 \mathrm{HDu}$. In the comparison between T2 and T3, the minimum, maximum and mean Hausdorff Distances were $0.000000,0.025616$ and $0.000347 \mathrm{HDu}$, respectively. In the current study, the image artifacts caused by metal orthodontic accessories did not compromise the accuracy of the 3D model superimposition. Color-coded maps of overlaid structures complemented the computed Hausdorff Distances and demonstrated a precise fusion between the data sets.
\end{abstract}

Descriptors: Cone-Beam Computed Tomography; Orthodontics; Artifacts.

\section{Introduction}

Cone Beam Computed Tomography (CBCT) has great diagnostic potential in all areas of dentistry, especially for cases in which its use has been substantiated to enhance diagnosis and treatment planning and for cases in which its benefits exceed the risks. ${ }^{1}$

Some CBCT scanners are able to deliver lower radiation doses than that of conventional Multi Slice Computed Tomography (MSCT) scanners when administered over similar volumes. ${ }^{2,3}$ It may therefore be speculated that the number of CBCT scans performed will be massively increased in the near future. However, if metal is present in the field of view (FOV), X-ray imaging techniques are always prone to producing artifacts. Streaking artifacts in Computed Tomography $(\mathrm{CT})$ images are 
caused by the attenuation characteristics of metal within the FOV and are generated mostly by metal restorations. ${ }^{4}$ Because of their higher atomic number, metals attenuate $\mathrm{X}$-rays in the diagnostic energy range much more than do soft tissue and bone. In fact, the X-ray beam is attenuated so strongly that almost no photons reach the detectors. The resultant effects show up in the CT images as pronounced dark and bright streaks, non-linear edge gradients, and sampling errors arising from the surface of a restoration. ${ }^{5,6}$ Streaking artifacts can be removed by interpolating raw data in the shadows of the metal object with adjacent raw data that does not contain the source of the artifact. The removed metal object is significantly scaled down in CT density and added back into the image. ${ }^{7}$ The results achieved with metal artifact reduction, however, still do not provide precise imaging of the occlusal relief of a tooth. ${ }^{8}$

Both MSCT and CBCT scanners use the filtered backprojection method as the basis for the $3 \mathrm{D}$ reconstruction process, thus following identical rules. As a consequence, metal artifacts are also present in CBCT images. ${ }^{9}$ The current literature, however, lacks research that specifically addresses this issue. ${ }^{10}$

Considering the potential of 3D CBCT voxelbased superimpositions, recent orthodontic papers have highlighted such methodology in follow-up longitudinal studies. ${ }^{11,12}$ However, if metals are present within the FOV, alterations in the voxels' grayscale may influence the superimposition procedure. ${ }^{13,14}$

Thus, the aim of the current study was to evaluate, through a $3 \mathrm{D}$ registration of unprocessed CBCT data, whether the image artifacts caused by orthodontic metal accessories have any effect on image superimposition.

\section{Methodology}

A dry human skull from the collection of the Anatomy Museum of Biomedical Sciences Institute at the University of São Paulo (USP) was used as the study object. All teeth were in good alignment and leveling. The mandible was related to the skull based on the position of the condyle in the fossa and on maximum occlusal interdigitation.

Orthodontic brackets (Orthometric Orthodon- tics $^{\mathrm{TM}}$, Marília, Brazil) were aligned and bonded on the dental buccal surfaces by using a conventional direct bonding technique with the Ortholink Bond System (Orthometric Orthodontics ${ }^{\mathrm{TM}}$, Marília, Brazil). The laboratory steps were performed at the Department of Orthodontics, School of Dentistry, USP.

The skull was positioned in the center of the scanning table in the same orientation as required for a live patient, with the assistance of vertical and horizontal light guides.

CBCT images of the dry skull area were acquired with an i-CAT Next Generation ${ }^{\mathrm{TM}}$ scanner (Imaging Sciences International Inc, Hatfield, USA) at the Department of Stomatology, School of Dentistry, USP. The first scan was taken without brackets (T1). A second scan was obtained with stainless steel brackets bonded on the upper and lower incisors, canines and premolars (10 brackets per arch) and with stainless steel buccal tubes bonded on the upper and lower molars (T2). For the third scan (T3), upper and lower $.019 " \times .025$ " stainless steel orthodontic arch wires (Orthometric Orthodontics ${ }^{\mathrm{TM}}$, Marília, Brazil) were inserted into the bracket slots.

The scanning parameters for imaging were $120 \mathrm{kVp}, 18.54 \mathrm{~mA}$, a scan time of 8.9 seconds, and a FOV of $17-\mathrm{cm}$ diameter $/ 22-\mathrm{cm}$ height. These settings produced a voxel size of $0.3 \mathrm{~mm}$. The T1, T2 and T3 scans followed the same protocol.

Raw data were collected and reconstructed into $3 \mathrm{D}$ volumes using the software from the CBCT scanner manufacturer. The reconstructed data were exported and saved as DICOM files without image modification (no brightness, contrast, or sharpness changes). Streaking artifacts in the T2 and T3 scans were not removed from the CBCT data. The volumetric data sets were post-processed with Mimics software, version 14.1 (Materialise Group, Leuven, Belgium), to convert DICOM files to the STL-format, which represents the 3D tomographic model in a mesh of non-uniform triangles. The STL files were imported into the open source software MeshLab (developed at the Computer Science Department of the University of Pisa, Italy; http://meshlab.sourceforge.net), version 1.3.0, on an iMac 27" $3.4 \mathrm{GHz}$, 16 Gb RAM, 2TB HD desktop computer (Apple Computer, Cupertino, USA). The registration proce- 
dure was carried out using the Meshlab software, which includes a subsystem for the alignment of 3D meshes, allowing for the registration of many different raw range maps. The software also includes a measuring tool, which allows for linear measurements between points of the displayed meshes. The registration processes were performed by the same operator.

The first procedure was the coarse registration between the T1, T2, and T3 meshes, which was performed in three pairs:

- T1-T2,

- T1-T3 and

- T2-T3.

After the meshes had been superimposed in a satisfactory initial position, the fully automated voxel-based alignment was completed, and the fine registration was performed. The fine registration was divided into three steps as follows.

In the first step, the registration was carried out using an Iterative Closest Point (ICP) algorithm, which automatically aligned the T1-T2, T1-T3 and T2-T3 pairs of meshes based on the corresponding nearest points. This method was used to find the best rigid transformation that brought the points of a first mesh onto the corresponding points of a second one, within the same reference space.

The second step involved Global Alignment, which is also known as multiview registration. In this stage, the distribution of any error in the alignment is detected among all the alignments that may occur to avoid the biased accumulation of errors. After the completion of registration, the number of corresponding points was counted, and the minimum, maximum and mean distances between the data points of the three pairs of superimposed meshes were calculated to assess the registration error.

Each pair of meshes was superimposed twice, changing the mesh that was considered as a reference. Therefore, considering T1, T2 and T3, the following superimpositions were performed:

- T1-T2 and T2-T1;

- T1-T3 and T3-T1;

- T2-T3 and T3-T2.
Therefore, six superimpositions were carried out for the three pairs of meshes.

The third and last step was the calculation of absolute units, which, in this study, was expressed in Hausdorff Distance units $(\mathrm{HDu})$. Surface distances between superimposed meshes were calculated, and color-coded 3D models were generated. Thus, the distance between surfaces at any location could be evaluated through quantitative (computed Hausdorff Distances) and qualitative (obtained color-coded images) appraisals. The image artifacts caused by orthodontic appliances were visually displayed and quantified using these 3D color maps. Structures not affected by metal artifacts during the superimposition process were coded in red, while the image artifacts caused by metal accessories were coded in yellow, green and blue.

\section{Results}

The color maps indicated the presence of image artifacts (yellow, green and blue) between the overlaid structures. The absence of any change received a Hausdorff Distance of 0.00 and was indicated in red, as shown in the MeshLab color scale (Figures 1 through 3). The color maps specified the color display range (minimum, maximum) of the surface distances. Any values that were lower than the minimum or higher than the maximum were mapped to the corresponding color. Any values between the minimum and maximum values were mapped from red to blue.

The minimum, maximum and mean Hausdorff

Table 1 - Hausdorff Distance calculations (C1 and C2) and minimum, maximum and mean Hausdorff Distances computed between meshes.

\begin{tabular}{c|c|c|c}
\hline $\begin{array}{c}\text { Hausdorff Distance } \\
\text { calculation (C) }\end{array}$ & $\begin{array}{c}\text { Minimum } \\
\text { distance } \\
\text { (HDu) }\end{array}$ & $\begin{array}{c}\text { Maximum } \\
\text { distance } \\
\text { (HDu) }\end{array}$ & $\begin{array}{c}\text { Mean } \\
\text { distance } \\
(\mathrm{HDu})\end{array}$ \\
\hline T2 on T1 (C1) & 0.000000 & 0.049280 & 0.002497 \\
\hline T1 on T2 (C2) & 0.000000 & 0.027239 & 0.002259 \\
\hline T3 on T1 (C1) & 0.000000 & 0.047440 & 0.002585 \\
\hline T1 on T3 (C2) & 0.000000 & 0.027365 & 0.002273 \\
\hline T3 on T2 (C1) & 0.000000 & 0.025616 & 0.000347 \\
\hline T2 on T3 (C2) & 0.000000 & 0.021818 & 0.000279 \\
\hline
\end{tabular}




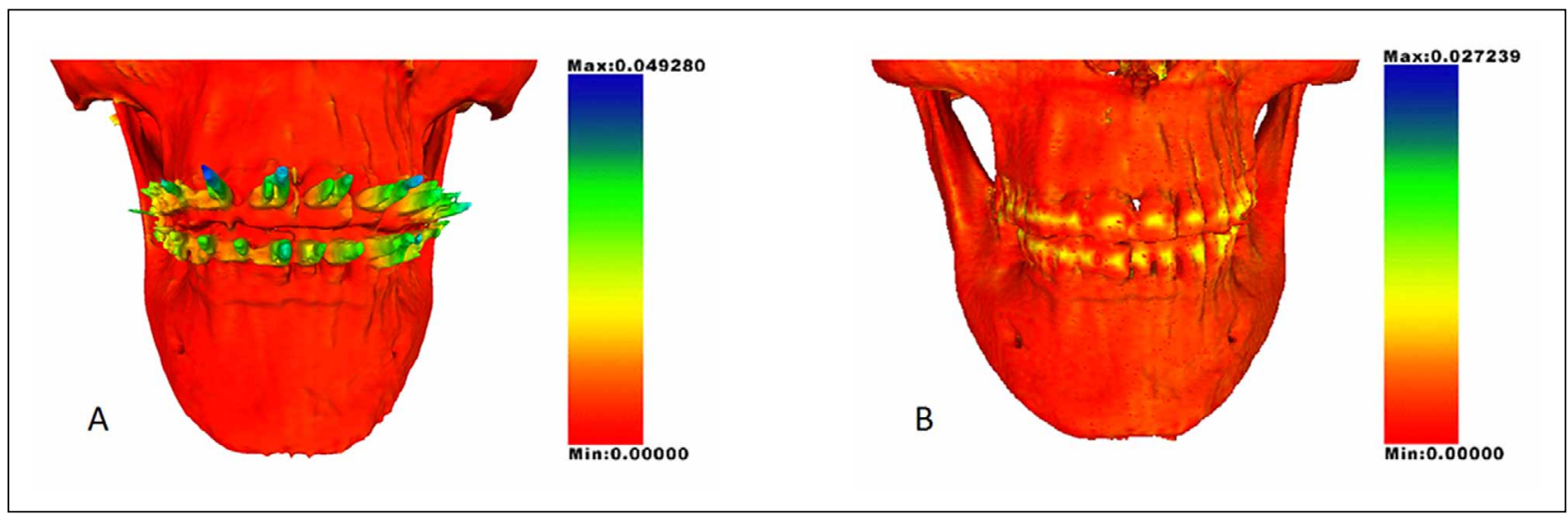

Figure 1 - Superimpositions between T1 and T2 meshes, with calculations 1 (A) and 2 (B).

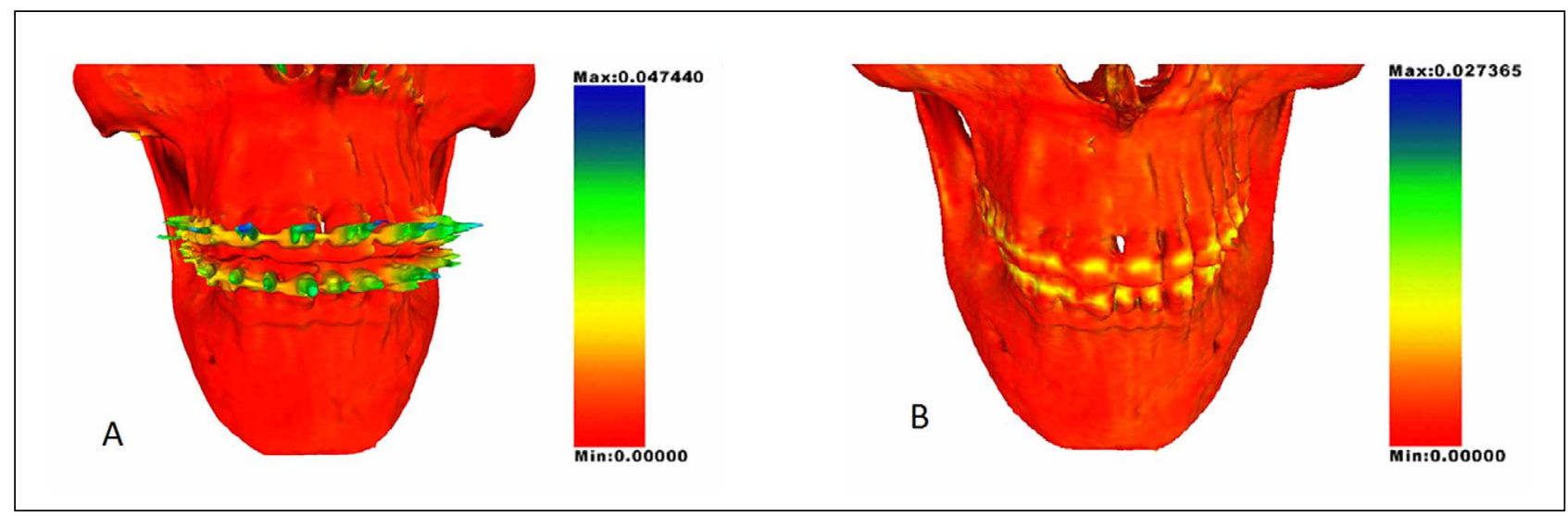

Figure 2 - Superimposition between T1 and T3 meshes, with calculations 1 (A) and 2 (B).

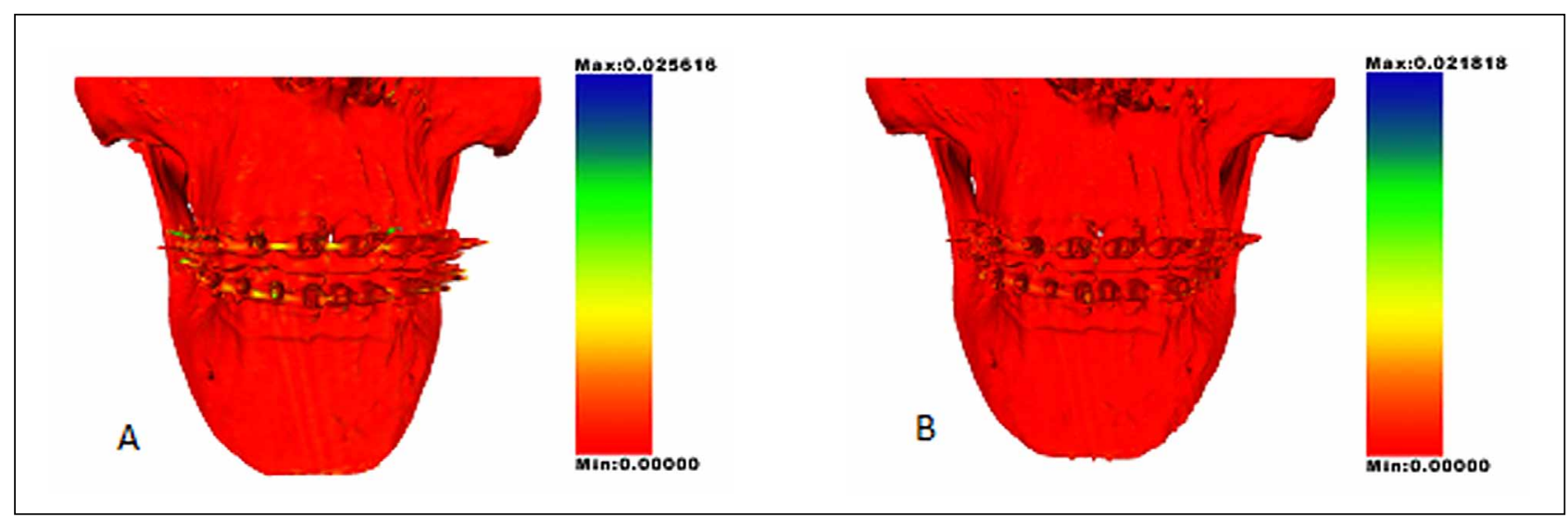

Figure 3 - Superimpositions between T2 and T3 meshes, with calculations 1 (A) and 2 (B).

Distance units $(\mathrm{HDu})$ computed between the corresponding data points of the T1 and T2 meshes (T1 as a reference, named calculation 1 ) were 0.000000 , 0.049280 and $0.002497 \mathrm{HDu}$, respectively. Consid- ering the T2 mesh as a reference (named calculation 2), the minimum, maximum and mean distances were $0.000000,0.027239$ and $0.002259 \mathrm{HDu}$, respectively (Table 1 ). 
The superimposition of the T2 on the control T1 mesh highlights the image artifacts of the brackets, which received yellow, green and blue colors. The occurrence of such artifacts, however, did not compromise the visualization and identification of bone and dental contours, as shown in red, when T1 was superimposed on the T2 mesh (Figure 1).

Regarding the comparison between the $\mathrm{T} 1$ and T3 meshes and considering T1 as a reference (calculation 1), the minimum, maximum and mean Hausdorff Distances were 0.000000, 0.047440 and $0.002585 \mathrm{HDu}$, respectively. When the T3 mesh was adopted as a reference (calculation 2), the minimum, maximum and mean Hausdorff Distances were $0.000000,0.027365$ and 0.002273 , respectively (Table 1$)$. The color-coded models displayed the image artifacts caused by orthodontic metal brackets and wires and provided the appropriate visualization of bone and dental contours during the superimposition process (Figure 2).

The superimposition between the T2 and T3 images highlighted the artifacts caused by the stainless steel orthodontic arch wires, which were coded in yellow (Figure 3). The minimum, maximum and mean distances, when $\mathrm{T} 2$ was considered as a reference (calculation 1), were $0.000000,0.025616$ and $0.000347 \mathrm{HDu}$, respectively. When the T3 mesh was considered as a reference (calculation 2), the minimum, maximum and mean Hausdorff Distances were $0.000000,0.021818$ and $0.000279 \mathrm{HDu}$, respectively (Table 1 ).

\section{Discussion}

Despite causing significant image artifacts in CBCT images, conventional stainless steel brackets and wires are widely used in orthodontic and orthognathic surgery patients and were therefore chosen for analysis in the present study. Considering the accuracy and reliability of voxel-based registration, ${ }^{15,16}$ our aim was to use this methodology to evaluate the effects of streaking artifacts caused by common orthodontic appliances on a 3D model superimposition.

In a previous study performed by Sanders et al. ${ }^{17}$ artifacts generated by different types of orthodontic brackets in CBCT images from a NewTom 3G scan- ner (AFP Imaging, Elmsford, NY) were evaluated. The authors compared human skulls with ceramic, plastic, stainless steel and titanium brackets with a control without brackets. Despite the fact that artifacts were more consistently associated with stainless steel and titanium brackets, the data reported were limited mainly to the axial plane and at the dental level. No evaluation regarding 3D volumetric data or bone structures was performed.

The determination of accuracy in a registration procedure is always a difficult task. To guarantee an effective correspondence between each pair of 2 data sets in the current study, the 2 sets were roughly aligned before starting the registration procedure. These first alignments were performed manually, in agreement with other similar methodologies..$^{15,16}$ The final alignment consisted of a fully automated voxel-based registration method, using the MeshLab software.

The Hausdorff Distance unit $(\mathrm{HDu})$ calculations between corresponding points of the three pairs of meshes were visually complemented by the generated color-coded models, which also allowed for a $3 \mathrm{D}$ visualization of surface distances. The image artifacts caused by orthodontic accessories were gradually color-coded according to increasing outward distance from the dental surfaces. The artifacts closer to the dental buccal surfaces were coded in yellow, while those farther away were coded in blue. These artifacts were more evident when the T2 and T3 were superimposed onto the T1 mesh because T1 did not have any orthodontic accessories. Because orthodontic appliances were not present in the T1 mesh, different colors were attributed to metal artifacts when T1 was used as the reference for superimposition with T2 and T3. This fact also explains the higher Hausdorff Distance ( $\mathrm{HDu})$ values observed when the T2 and T3 meshes were superimposed on the T1 mesh.

In each of the comparisons (T1-T2, T1-T3 and T2-T3), the mesh superimpositions and distance calculations were performed twice (calculations 1 and 2), changing the mesh used as a reference. Although the same skull was used for the three CBCT scans, the presence of stainless steel brackets (T2) and wires attached into the bracket slots (T3) led to 
numerical differences between calculations 1 and 2 within the same pairs of meshes (Table 1). The computed differences did not compromise the visual identification of either bone or dental contours, which were coded in red. These qualitative findings are apparent in comparisons between Figures 1 (A and $\mathrm{B}), 2$ (A and $\mathrm{B}$ ), and 3 ( $\mathrm{A}$ and $\mathrm{B}$ ).

The resulting artifacts did not compromise the visual identification of dental surfaces near the orthodontic brackets. The imaging quality of the dentition remained satisfactory, despite the presence of metal accessories bonded on the buccal surfaces of the upper and lower teeth. In contrast, Nkenke et al. ${ }^{8}$ found significant differences when performing the fusion between optical 3D and CT imaging of plaster casts with and without metal restorations. The differences between these data were considered statistically significant, and the accuracy of the fusion of 3D CT surface data and optical 3D imaging was significantly reduced by the occurrence of metal artifacts.

In real clinical situations, soft tissues cover the upper and lower jaws, and facial muscles and skin are also present. Because a dry skull was used in our study, the results may not directly correlate with CBCT images obtained from patients. Furthermore, the current study employed an experimental model that was not affected by any kind of movement. In daily practice, however, image acquisition can be influenced by accidental and/or physiological move-

\section{References}

1. Kapila S, Conley RS, Harrell Jr WE. Current status of cone beam computed tomography imaging in orthodontics. Dentomaxillofac Radiol. 2011 Jan;40(1):24-34.

2. Ludlow JB, Davies-Ludlow LE, Brooks SL, Howerton WB. Dosimetry of 3 CBCT devices for oral and maxillofacial radiology: CB Mercuray, NewTom 3G and i-CAT. Dentomaxillofac Radiol. 2006 Jul;35(4):219-26.

3. Ludlow JB, Ivanovic M. Comparative dosimetry of dental CBCT devices and 64-slice CT for oral and maxillofacial radiology. Oral Surg Oral Med Oral Pathol Oral Radiol Endod. 2008 Jul;106(1):106-14.

4. Tohnak S, Mehnert AJH, Mahoney M, Crozier S. Dental CT metal artefact reduction based on sequential substitution. Dentomaxillofac Radiol. 2011 Mar;40(3):184-90. ments of the patient during scanning. ${ }^{18}$ These factors may lead to slight differences between our experimental results and more realistic clinical images; thus, future studies are necessary to evaluate specific clinical situations. The results of this experimental study indicated that metal artifacts did not alter the dental and bone volumetric image contours, although such artifacts were clearly visualized in the T2 and T3 meshes. The Hausdorff Distances between corresponding points were calculated for the total skull data because no previous segmentation had been performed. Although the computed distances differed between the superimposed meshes, the accuracy of the procedure was not affected by the presence of orthodontic accessories. The reported results suggest that the beam hardening caused by metal orthodontic appliances in the present study was not close and/or strong enough to influence the 3D superimpositions performed.

\section{Conclusions}

The present findings elucidated that metal orthodontic appliances generate artifacts in CBCT images; however, these artifacts did not compromise the superimposition of the $3 \mathrm{D}$ models in this study. The results of the voxel-based superimposition were qualitatively complemented by color maps, which showed a precise fusion of the volume overlap between the data sets.

5. Svendsen P, Quiding L, Landahl I. Blackout and other artifacts in computed tomography caused by fillings in teeth. Neuroradiology. $1980 \mathrm{Jul} ; 19(5): 229-34$.

6. Odlum O. A method of eliminating streak artifacts from metal dental restorations in CTs of head and neck cancer patients. Spec Care Dentist. 2001 Mar-Apr;21(2):72-4.

7. Vannier MW, Hildebolt CF, Conover G, Knapp RH, Yokoyama-Crothers N, Wang G. Three-dimensional dental imaging by spiral CT. A progress report. Oral Surg Oral Med Oral Pathol Oral Radiol Endod. 1997 Nov;84(5):561-70.

8. Nkenke E, Zachow S, Benz M, Maier T, Veit K, Kramer M, et al. Fusion of computed tomography data and optical 3D images of the dentition for streak artefact correction in the simulation of orthognathic surgery. Dentomaxillofac Radiol. 2004 Jul;33(4):226-32. 
9. Schulze RKW, Berndt D, d'Hoedt B. On cone-beam computed tomography artifacts induced by titanium implants. Clin Oral Implants Res. 2010 Jan;21(1):100-7.

10. Schulze R, Heil U, Grob D, Bruellmann DD, Dranischnikow E, Schwanecke U, et al. Artefacts in CBCT: a review. Dentomaxillofac Radiol 2011 Jul;40:265-73.

11. Cevidanes LHS, Motta A, Proffit WR, Ackerman JL, Styner M. Cranial base superimposition for 3-dimensional evaluation of soft-tissue changes. Am J Orthod Dentofacial Orthop. 2010 Apr;137(4 Suppl):S120-9.

12. Heymann GC, Cevidanes LHS, Cornelis M, De Clerck HJ, Tulloch JFC. Three-dimensional analysis of maxillary protraction with intermaxillary elastics to miniplates. Am J Orthod Dentofacial Orthop. 2010 Feb;137(2):274-84.

13. Katsumata A, Hirukawa A, Noujeim M, Okumura S, Naitoh M, Fujishita M, et al. Image artifact in dental cone-beam CT. Oral Surg Oral Med Oral Pathol Oral Radiol Endod. 2006 May;101(5):652-7.

14. Katsumata A, Hirukawa A, Okumura S, Naitoh M, Fujishita $\mathrm{M}$, Ariji E, et al. Effects of image artifacts on gray-value density in limited-volume cone-beam computerized tomography.
Oral Surg Oral Med Oral Pathol Oral Radiol Endod. 2007 Dec;104(6):829-36.

15. Cevidanes LHS, Bailey LJ, Tucker GR Jr, Styner MA, Mol A, Phillips CL, et al. Superimposition of 3D cone-beam CT models of orthognathic surgery patients. Dentomaxillofac Radiol. 2005 Nov;34(6):369-75.

16. Swennen GRJ, Mollemans W, De Clercq C, Abeloos J, Lamoral P, Lippens F, et al. A Cone-Beam Computed Tomography Triple Scan Procedure to Obtain a Three-Dimensional Augmented Virtual Skull Model Appropriate for Orthognathic Surgery Planning. J Craniofac Surg. 2009 Mar;20(2):297-307.

17. Sanders MA, Hoyjberg C, Chu CB, Leggitt VL, Kim JS. Common orthodontic appliances cause artifacts that degrade the diagnostic quality of CBCT images. J Calif Dent Assoc. 2007 Dec;35(12):850-7.

18. Cremonini CC, Dumas M, Pannuti CM, Neto JBC, Cavalcanti MGP, Lima LA. Assessment of linear measurements of bone for implant sites in the presence of metal artefacts using cone beam computed tomography and multislice computed tomography. Int J Oral Maxillofac Surg. 2011 Aug;40(8):845-50. 